\title{
Appendix S5
}

\section{How $N$ affects the model parameters and simulation results}

Since $\alpha$ is determined from $\alpha+\beta=2$ and $\alpha / \beta=V_{+} / V_{-}$, and $\Delta r$ from $\Delta r=\frac{1}{2}\left(d_{\text {bear }}-d_{\text {bull }}\right)$, both $\alpha$ and $\Delta r$ are not dependent on the number of agents (denoted by $N$ ). On the other hand, $\Delta R$ is calculated from the linear relation between $\Delta R$ and $\Delta r$, which is obtained through the simulation of the model. As shown below in Table 1, the slope of the linear relation increases as $N$ grows, and it leads to a larger magnitude of $\Delta R$ for larger $N$. In the main body of the paper, for simplicity, $\Delta R$ is approximated to an integer in the simulation for each index. To investigate how $N$ affects the simulation results, however, $\Delta R$ should be more accurate, and it is now corrected to one decimal place. The influence of $N$ on $L(t)$ is displayed in Fig. 1. The amplitude of $L(t)$ increases with $N$, but gradually converges for larger $N$. For $A(t)$ and $P(|r(t)|>x)$, the cases are similar.

Table 1: The values of $\Delta R$ and the relations between $\Delta R$ and $\Delta r$ for different $N$ for the $\mathbf{S} \& \mathbf{P} 500$ and Shanghai indices.

\begin{tabular}{|c|c|cc|cc|}
\hline \multirow{2}{*}{$N$} & relation of $\Delta R$ and $\Delta r$ & \multicolumn{2}{|c|}{ S\&P 500 Index } & \multicolumn{2}{|c|}{ Shanghai Index } \\
& & $\Delta r$ & $\Delta R$ & $\Delta r$ & $\Delta R$ \\
\hline 1250 & $\triangle R=12.4 \Delta r$ & 0.067 & 0.8 & -0.043 & -0.5 \\
2500 & $\triangle R=17.6 \Delta r$ & 0.067 & 1.2 & -0.043 & -0.8 \\
5000 & $\triangle R=25.4 \Delta r$ & 0.067 & 1.7 & -0.043 & -1.1 \\
10000 & $\triangle R=38.2 \Delta r$ & 0.067 & 2.6 & -0.043 & -1.6 \\
20000 & $\triangle R=61.6 \Delta r$ & 0.067 & 4.1 & -0.043 & -2.6 \\
\hline
\end{tabular}

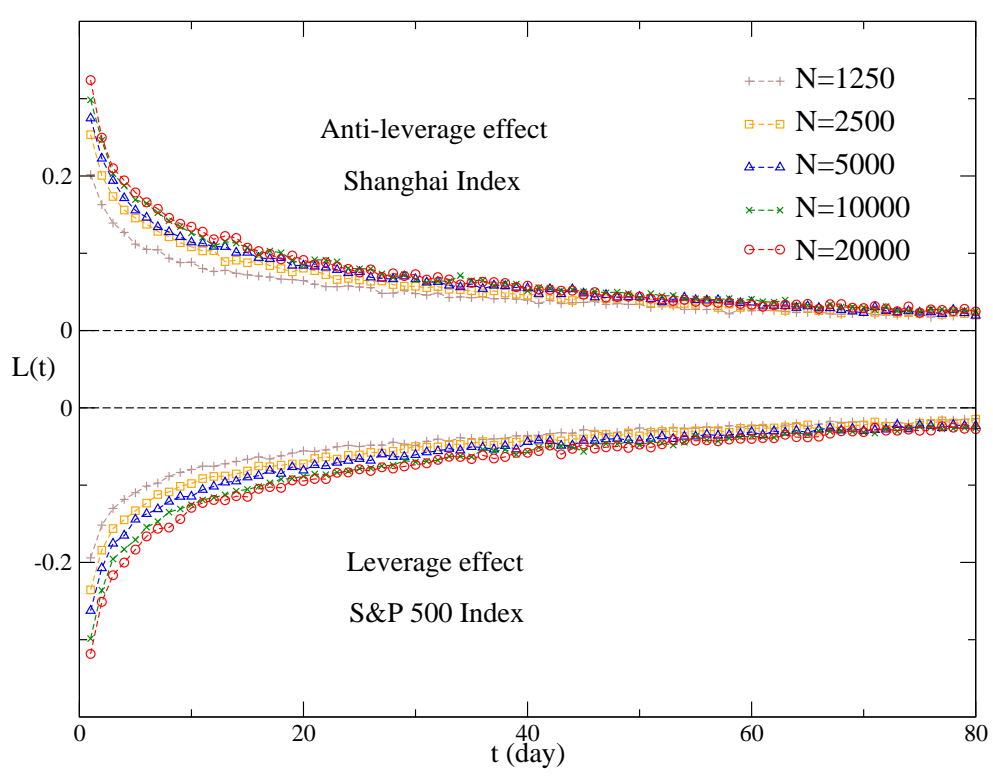

Figure 1: $L(t)$ from the simulations with different $N$ for the S\&P 500 and Shanghai indices. The S\&P 500 Index exhibits the leverage effect, and the Shanghai Index shows the anti-leverage effect. For each $N$, the simulation is performed 100 times for average. 